\title{
YAPI Promotes Tumor Invasion and Metastasis in Nasopharyngeal Carcinoma with Hepatitis B Virus Infection
}

This article was published in the following Dove Press journal: OncoTargets and Therapy

Zeli Huang, ${ }^{1, *}$ Bojin Su, ${ }^{2, *}$ Fang Liu, (D) ${ }^{3, *}$ Ning Zhang,' Yilong Ye, ${ }^{4}$ Yang Zhang,' Zhenghe Zhen,' Shaoqiang Liang,' Shaobo Liang,' Lushi Chen,' Weijun Luo,' François $X$ Claret, ${ }^{5,6}$ Ying Huang, ${ }^{7,8}$ Tao $\mathrm{Xu}^{\prime}$

'Department of Radiation Oncology, Cancer Center, First People's Hospital of Foshan, Foshan 528000, Guangdong Province, People's Republic of China; ${ }^{2}$ Department of Pathology, The Third Affiliated Hospital of Sun Yat-sen University, Guangzhou 510630, Guangdong Province, People's Republic of China; ${ }^{3}$ Department of Pathology, First People's Hospital of Foshan, Foshan 528000, Guangdong Province, People's Republic of China; ${ }^{4}$ Department of Infection, First People's Hospital of Foshan, Foshan 528000 , Guangdong Province, People's Republic of China; ${ }^{5}$ Department of Systems Biology, The University of Texas MD Anderson Cancer Center, Houston, TX 77030, USA; ${ }^{6}$ Experimental Therapeutics Academic Program and Cancer Biology Program, The University of Texas Graduate School of Biomedical Sciences at Houston, Houston, TX 77030, USA; ${ }^{7}$ Department of Radiation Oncology, Cancer Center, Sun Yat-sen University, Guangzhou 510080, Guangdong Province, People's Republic of China; ${ }^{8}$ State Key Laboratory of Oncology in South China, Collaborative Innovation Center for Cancer Medicine, Guangdong Key Laboratory of Nasopharyngea Carcinoma Diagnosis and Therapy, Sun Yat-sen University Cancer Center, Guangzhou 510060 People's Republic of China

*These authors contributed equally to this work

Correspondence: Ying Huang Department of Radiation Oncology, Cancer Center, Sun Yat-sen University, Guangzhou 510080, Guangdong Province, People's Republic of China

Email huangying@sysucc.org.cn

Tao Xu

Department of Radiation Oncology, Cancer Center, First People's Hospital of Foshan, Foshan 528000, Guangdong Province, People's Republic of China Email asian.you@I63.com
Introduction: Nasopharyngeal carcinoma (NPC) patients with HBsAg $(+)$ commonly present with high frequencies of distant metastasis and poor survival rate; however, the mechanism has not been elucidated.

Materials and Methods: We analyzed the yes-associated protein 1 (YAP1) expression between HBsAg (+) and HBsAg (-) of NPC patients, then analyzed the relationship of YAP1 with survival. We further explored the anti-tumor role in NPC cell lines using YAP1 siRNA technique, and checked whether YAP1 regulatesepithelial-mesenchymal transition ( EMT). The relationship between HBV X protein $(\mathrm{HBx})$ and YAP1 was also tested using DualLuciferase reporter assay. Finally, we explored anti-YAP1 to inhibit tumor metastasis using the xenograft mice model.

Results: In the current study, we found that YAP1 expression was higher in HBsAg $(+)$ samples than in the HBsAg (-) samples, as a clinical signature, suggesting that YAP1 could be used as a prognostic factor for NPC. Our results showed that the HBx could regulate YAP1, further promoting cellular invasiveness through EMT. Anti-YAP1 can also decrease metastasis in vivo.

Conclusion: Our findings suggest that YAP1 is a promising prognostic factor in NPC and could be used as a potential treatment target for NPC with HBV infection.

Keywords: YAP1, hepatitis B, HBx, nasopharyngeal carcinoma, metastasis

\section{Introduction}

Nasopharyngeal carcinoma (NPC) is a malignant tumor occurring in the nasopharyngeal cavity which possesses various distinctive clinical characteristics, including the highest incidence rate among Cantonese in Southern China, high responsiveness to radiotherapy and chemotherapy, a strong link to Epstein-Barr virus (EBV), and is prone to locoregional recurrence or metastasis. ${ }^{1-5}$ Hepatitis B virus (HBV) infection is also common in Southern China, with 70-95\% of the population showing past or present serological evidence of $\mathrm{HBV}$ infection. It is believed that $\mathrm{HBV}$ is related to the development of liver chronic cirrhosis and eventually malignant carcinoma., 6 Liu et al firstly demonstrated hepatitis B surface antigen (HBsAg) as an independent, negative prognostic factor in the NPC cohort, through large clinical data analysis. $^{8}$ In our previous study, we found that HBsAg $(+)$ patients, accounting for $10.3 \%$ of the NPC cohort, with young males seemingly more susceptible to infection, had a poor survival rate if the HBsAg $(+)$ patient presented with a high level of pre-infected EBV DNA. ${ }^{9}$ However, there is no clear explanation as to why 
NPC patients with HBV infection exhibit a poor prognosis, and presently published data are not very clear.

The Hippo pathway has been shown to be critical in tumorigenesis, and recent data from The Cancer Genome Atlas (TCGA) strongly support that this pathway affects overall cancer survival. ${ }^{10}$ Yes-associated protein 1 (YAP1), a novel and significant protein in the Hippo pathway, is critical in tumor cell proliferation and invasion. ${ }^{11-13}$ Increasing evidence support that epithelial-mesenchymal transition (EMT) was required for epithelial cells to acquire malignant ability. Shao et al's findings showed YAP1 can trigger transcriptional regulation of EMT as a significant component of oncogenic RAS signaling. ${ }^{11}$ A study from Omori et al showed that co-overexpression of YAP1, SOX2 and CD44v9 was closely associated with poor prognosis for head and neck cancer, and these molecular might induce cancer stem cell, which was related to EMT and angiogenesis. ${ }^{12}$ In another study, YAP1 was proven to drive the metaplastic transformation of squamous cell carcinoma to spindle cell carcinoma and its mechanism was that YAP1 could induce ZEB1 and EMT. ${ }^{13}$ Recently, our analysis of the signature of key distant metastasis proteins, using a proteomic approach with the reverse phase protein array (RPPA) platform, showed that YAP1 protein was highly expressed in metastatic patients. ${ }^{14}$ Furthermore, we analyzed and compared differential expression in NPC, with or without HBsAg, and found that YAP1 was highly expressed in HBsAg $(+)$ patients. Therefore, we hypothesize that YAP1, as an oncogene, acts as a distant metastasis promoter in HBsAg $(+)$ NPC. Understanding how YAP1 promotes metastasis could give a hint for new therapeutic approaches in NPC treatment.

In the current study, we explore the regulation mechanisms of YAP1 in NPC with HBV infection. Hepatitis $\mathrm{B}$ virus $\mathrm{X}$ protein $(\mathrm{HBx})$ encoded by $\mathrm{HBV}$ is essential in primary hepatocellular carcinoma. ${ }^{15,16}$ This is the first study to demonstrate that $\mathrm{HBx}$ can regulate YAP1 in NPC patients with HBV infection, and mechanically, YAP1 promotes NPC cell invasion through EMT. Our findings show that YAP1 could serve as a promising prognostic and treatment target for NPC with HBV infection.

\section{Materials and Methods}

\section{Cell Culture}

Human NPC cell lines (CNE1 and SUNE1) used in this study were obtained from the Cancer Center of Sun YatSen University (Guangzhou, China). The use of the cell lines was approved by the Ethics Committee of the First People's Hospital of Foshan (Guangdong Province, China). Cells were cultured using RPMI-1640 medium with $10 \%$ fetal bovine serum, $0.5 \%$ penicillin-streptomycin sulfate in $5 \%$ carbon dioxide humid incubator as in previous studies. ${ }^{17,18}$

\section{Ethics Statement}

Animal experiment was approved from the Institutional Animal Care and Use Ethics Committee of the First People's Hospital of Foshan, following guidelines for the welfare of the laboratory animals. All human samples were collected by following the protocols of the Ethics Committee of the First People's Hospital of Foshan (Guangdong Province, China). And all patients provided written informed consent, in accordance with the Declaration of Helsinki. According to ethical and legal standards, all patient tissue samples and clinical data were anonymously processing.

\section{RPPA Data and ONCOMINE Database Analysis}

We compared the difference in YAP1 expression between HBsAg (+) and (-) groups based on proteomics analysis using an RPPA platform, which has been described previously. ${ }^{14}$ The ONCOMINE database (www.onco mine.com/nasophryngeal cancer) was used to analyze yap gene expression with clinical TNM staging.

\section{Clinical Data and Tissue Samples}

We collected two independent databases for the study of YAP1, including 6 normal nasopharyngeal mucosa samples and 25 NPC diagnosed patients with HBsAg (+). To further confirm YAP1 as a prognostic factor for NPC survival, 70 formalin-fixed paraffin-embedded (FFPE) patient specimens (35 metastatic cases and 35 non-metastatic) were analyzed for YAP1 expression. All clinical data of patients are summarized in Table 1. Clinical staging was conducted according to the staging system of the American Joint Committee on Cancer (AJCC, 7th edition).

\section{Plasmid and siRNA Transfection}

Cells $\left(1 \times 10^{5}\right.$ cells/well $)$ were seeded into 6-well plates 24 $\mathrm{h}$ before transfection. Then, $120 \mathrm{nM} \sim 160 \mathrm{nM}$ of YAP1 siRNA or control siRNA oligonucleotides (with/without $1 \sim 4 \mu \mathrm{g}$ of HBx plasmid), or $6 \mu \mathrm{g}$ of Myc-YAP1-pcDNA plasmid were transfected using Lipofectamine 2000 (Invitrogen, Carlsbad, CA, USA) protocol, transfected cells were culture in RPMI-1640 with 10\% FBS and no 
penicillin-streptomycin for $48 \mathrm{~h}$. The siControl and YAP1 siRNA (YAP1-homo-1355) were purchased from Shanghai GenePharma Co., Ltd.

\section{Establishment of shRNA-Stable Cells}

YAP1 shRNA oligonucleotides were cloned into a retrovirus system (Clontech, Mountain view, CA, USA) to establish YAP1 knockdown stable cell line. In short, co-transfected with a YAP1 shRNA-vector DNA with vectors $\mathrm{pLP}$ and pVSVG into the 293T-packaging cell. Forty-eight hours after transfection, the supernatant was collected, then further to infect the target NPC cells. Stable clones were selected with 14 days of $0.6 \mu \mathrm{g} / \mathrm{mL}$ puromycin treatment, and further confirmed the positive clone by Western blot. At last, positive clones were maintained in $0.2 \mu \mathrm{g} / \mathrm{mL}$ puromycin culture condition.

\section{Cell Viability Assay and Colony Formation}

We evaluate relative cell viability using the 3-(4,5-dimethylthiazol-2-yl)-2,5-diphenyltetrazolium bromide (MTT) assay. Briefly, NPC cells were plated into 96well plates overnight, $48 \mathrm{~h}$ after si-YAP1 with or without HBx plasmid transfection. The spectrophotometric absorbance of the samples was read after the MTT labeling reagent was added. The IC50 values were evaluated by using GraphPad Prism 4 (GraphPad Software, La Jolla, CA, USA).

To analysis whether YAP1 or HBx affect growth ability, SUNE1 and CNE1 cells (400 cells per well) were treated with siYAP1 with or without HBx transfection 12 $\mathrm{h}$ after cells seeded into 6-well plates. Cells were fixed, stained after 10 days of culturing. And colonies (50 or more cells) were counted with an inverted microscope.

\section{Wound-Healing Assay}

siYAP1 and HBx DNA plasmid transfection were performed $12 \mathrm{~h}$ after SUNE1 and CNE1 cells were plated into 6-well plates. The next day, a scratch (across the center of the cell plate) was made by using a sterile 200$\mu \mathrm{L}$-pipette tip, and then incubated for $24 \mathrm{~h}$ or $48 \mathrm{~h}$. Images of the cells were acquired and the migration distance was measured using phase-contrast optics. The scratch wound widths were defined as the percentage of cells that could migrate into the scratch area.

\section{Cell Invasion Assays}

We evaluated the tumor cell invasion ability by using a Transwell Chamber (Corning Incorporated, Life Sciences, USA) with $8-\mu \mathrm{m}$ pores coated with Matrigel
(BD, Biosciences, USA). Cells were treated with siYAP with or without $\mathrm{HBx}$ infection $24 \mathrm{~h}$ before plated into the upper chamber, and a higher percentage of fetal bovine serum $(10 \%)$ was placed in the lower chamber. The plates were incubated for $24 \mathrm{~h}$, then fixed with $10 \%$ methanol and $0.1 \%$ crystal violet for $15 \mathrm{~min}$ at room temperature, the images were acquired for further analysis.

\section{Western Blot}

Total cell lysates were extracted and then added on $10 \%$ SDS-PAGE to separate the targeting protein, next, transferred to nitrocellulose polyvinylidene difluoride membranes, then incubated with primary polyclonal antibodies overnight (anti-YAP1 \#8418,1:1000 dilution, anti-Snail, \#3879,1:800 dilution, anti-E-cadherin \#14472,1:1000 dilution, anti- N-cadherin \#13116,1:1000 dilution, anti-LATS1 \#3477,1:700 dilution, anti-MST1 \#14946,1:700 dilution; Cell Signaling Technology, USA). The next day, it was probed with secondary antibodies before detecting for the blots, the later one was using the chemiluminescence system (Bio-Rad, CA, USA).

Table I Patients Characteristics in the Current Study

\begin{tabular}{|c|c|c|c|}
\hline \multirow[t]{2}{*}{ Characteristics } & \multicolumn{2}{|c|}{ YAPI Expression } & \multirow[t]{2}{*}{$\mathbf{P}$} \\
\hline & Low $(N=40)$ & High $(N=30)$ & \\
\hline \multicolumn{4}{|l|}{ Sex } \\
\hline Male & $36(90.0)$ & $23(76.7)$ & 0.118 \\
\hline Female & $4(10.0)$ & $7(23.3)$ & \\
\hline \multicolumn{4}{|l|}{ Age (years) } \\
\hline$<50$ & $22(55.0)$ & $15(50.0)$ & 0.431 \\
\hline$\geq 50$ & $18(45.0)$ & $15(50.0)$ & \\
\hline \multicolumn{4}{|l|}{ T category } \\
\hline TI & I (2.5) & I (3.3) & 0.895 \\
\hline T2 & II (27.5) & $6(20.0)$ & \\
\hline T3 & $10(25.0)$ & $9(30.0)$ & \\
\hline T4 & $18(45.0)$ & I4 (46.7) & \\
\hline \multicolumn{4}{|l|}{$\mathrm{N}$ category } \\
\hline No & 7 (I7.5) & $2(6.7)$ & 0.291 \\
\hline $\mathrm{NI}$ & $8(20.0)$ & $10(33.3)$ & \\
\hline N2 & $20(50.0)$ & $12(40.0)$ & \\
\hline N3 & $5(12.5)$ & $6(20.0)$ & \\
\hline \multicolumn{4}{|l|}{ TNM staging } \\
\hline I & $0(0.0)$ & $0(0.0)$ & 0.301 \\
\hline II & $5(12.5)$ & I (3.3) & \\
\hline III & $14(35.0)$ & $9(30.0)$ & \\
\hline IVA-B & $21(52.5)$ & $20(66.7)$ & \\
\hline
\end{tabular}




\section{RNA Extraction and Quantitative RT-PCR (qRT-PCR)}

Total RNA was extracted using RecoverAll ${ }^{\mathrm{TM}}$ Total Nucleic Acid Isolation Kit (Thermo Fisher Scientific, CA, USA), and collected using TRIzol (Ambion, Invitrogen, Carlsbad, CA, USA), by following the manufacturer's protocol. qRT-PCR was performed using Power SYBR-Green PCR master mix (Applied Biosystems Inc.) to detect mRNA levels of YAP1 and HBx; glyceraldehyde 3-phosphate dehydrogenase (GAPDH) was used to normalize the YAP1 and HBx values. The cycling instruction was as follows: $2 \mathrm{~min}$ of $50^{\circ} \mathrm{C}$ and $95^{\circ} \mathrm{C}$ for $10 \mathrm{~min}$ followed by 40 cycles at $95^{\circ} \mathrm{C}$ for $15 \mathrm{sec}$ and $1 \mathrm{~min}$ of annealing/extension at $60^{\circ} \mathrm{C}$. U6 snRNA or glyceraldehyde 3-phosphate dehydrogenase (GAPDH) was used to normalize the YAP1 and HBx values, respectively. Data were analyzed by the $2-\Delta \Delta \mathrm{Cq}$ method to determine relative changes in gene expression. The primers for PCR amplification were as follows: YAP forward, 5'-GG GTGTTCATCCATTCTC-3'; YAP reverse, 5'-CCCAGC ATCTTGTGTTTC-3'; HBx forward, 5'-GTGGGATGATG ACGACG-3', and HBx reverse, 5'-TACGAC CAGAG GCATACAGG-3'.

\section{Dual-Luciferase Reporter Assay}

SUNE1 cells were transfected using pGL3-Basic $(0.2 \mathrm{ng} /$ well) or reporter constructs of the YAP gene, as indicated (pGL3-348, $0.2 \mathrm{ng} /$ well). Relative luciferase activity results were obtained. SUNE1 cells were co-transfected with pGL3-348 (0.2 ng/well) and HBx expression plasmid (pCMV-HBx, 0.2 or $0.3 \mathrm{ng} /$ well) or control pcDNA $(0.2$ $\mathrm{ng} /$ well), respectively. The promoter activities of YAP were measured. All experiments were conducted in triple independent experiments. Primers for YAP promoter $\left(5^{\prime}-3^{\prime}\right)$ :

pGL3-348 forward CGGGGTACCCCAGGGCGAGC GGGTCACGT

\section{Immunohistochemical Analysis}

A total of 101 FFPE human specimens (6 normal nasopharyngeal mucosa samples, 25 NPC patients diagnosed with HBsAg (+), 70 NPC cases with intact clinical data and median 5-year follow-up) were analyzed with each specimen being de-paraffinized and sectioned. Briefly, the sections were incubated with primary antibodies against YAP1 (\#8418,1:200 dilution, Cell Signaling Technology, USA) and HBx (\#ab39716, 1:50 dilution, Abcam, MA, USA) at $4^{\circ} \mathrm{C}$ overnight, then incubated with the biotinylated secondary antibody, at last subjected to the Liquid $\mathrm{DAB}+$ Substrate Chromogen System (Dako, Carpinteria, CA). At least 500 tumor cells (NPC) or normal cells (nasopharyngeal inflammation) were counted for YAP1 and HBX expression evaluation in five representatives' high-power microscopy fields. Nuclear or cytoplasmic yellow or brown staining was considered positive expression. The proportion score represented the positive staining cells as follows: $0=$ less than $5 \% ; 1=5 \%$ to $25 \%$; $2=25 \%$ to $50 \% ; 3=$ greater than $50 \%$. The intensity score represented the estimated average staining as follows: $0=$ no staining; 1 = weak staining; $2=$ intermediate staining; $3=$ strong staining. The overall protein expression defined as the average of the proportion and intensity scores (ranges $=0,1,2-4$ and 6 to 9 , respectively). We determined negative $(-)$, weakly positive $(+)$, positive $(++)$ and highly positive $(+++)$ if the scores were $0,1,2-4$, and $6-9$, respectively.

\section{Xenograft Tumors in Nude Mice}

Animal experiments were performed following the guidelines of the Institutional Animal Care and Use Ethics Committee of the First People's Hospital of Foshan, Guangdong Province, China. Eight-week-old female $\mathrm{BALB} / \mathrm{c}$ nude mice were purchased from the Medical Experimental Animal Center of Guangdong Province (Guangzhou, China). To create a mice metastasis model, SUNE1 cells $\left(200 \mu \mathrm{L}\right.$ PBS containing $1 \times 10^{6}$ SUNE-1 cells) stably expressing shYAP1 or control shRNA (with or without HBx DNA plasmid, respectively) was injected into mice tail vein ( $n=6$ per group). Six weeks later, mice were euthanized, and then their lungs were collected. All lung samples were then paraffin-embedded, sectioned and stained for morphology, and also anti-Snail (\#sc271977, 1:100 dilution, Santa Cruz Biotechnology, USA), anti E-Cadherin (\#14472,1:200 dilution, Cell Signaling Technology, USA), anti-Vimentin (\#5741,1:200 dilution, Cell Signaling Technology, USA), anti-TWIST (\#ab49254, 1:80 dilution, Abcam, MA, USA) and anti-HBx (\#sc373980, 1:50 dilution, Santa Cruz Biotechnology, USA) immunohistochemistry staining was performed in these samples.

\section{Statistical Analysis}

For only two groups, Student's $t$-test was used for statistical analysis of the results. The characteristic of the high YAP1 and low YAP1 expression were compared using the chi-square test. Kaplan-Meier method was used to analyze 
actuarial survival rates and the Log rank test was used to compare differences in survival. Pearson's correlation coefficient was used to analyze the association between YAP and HBx expression. Two-tailed $\mathrm{P}$ values $<0.05$ were considered statistically significant. Statistical analysis was performed using SPSS software (version 16.0, IBM Corporation, Armonk, NY).

\section{Results}

\section{YAPI Expression in NPC Tissue with HBV Infection Correlated with Poor Prognosis}

We assessed YAP1 expression using IHC analysis, which turned out that YAP1 expression was much higher in NPC tissue $(n=25)$ than normal nasopharyngeal mucous epithelium $(n=6, p=0.035$, Figure $1 \mathrm{~A}$ and $C)$, which was consistent with results from the RPPA data reported previously. Due to the sample limitation, YAP1 expressions in HBsAg (+) NPC $(n=5)$ show no statistic significant compare to HBsAg (-) NPC $(n=65)(p=0.557$, Supplementary Figure $1 \mathrm{~A})$; however, we could see a tendency that a higher YAP1 expression in HBsAg $(+)$ NPC. Furthermore, we found that HBx expression positively correlated with YAP1 expression (Pearson correlation $=0.396 ; p=0.027$, Figure $1 C$ ), which gave us a clue that
YAP1 expression is positively related to $\mathrm{HBsAg}(+)$ NPC. What is more, survival analysis showed that high expression of YAP1 was likely associated with poor overall survival (39.7\% vs. $74.3 \%, p=0.043$, Figure 1D) and also show right survival tendency in progression-free survival and metastasisfree survival (Supplementary Figure 1B and C). The significance of positive finding suggests that YAP1 might also serve as an effective predictive biomarker for NPC.

\section{YAPI Inhibition Reduced Tumor Proliferation, Migration and Invasion}

Next, bio-function of YAP1 in NPC cells was explored. We analyzed whether RNAi-mediated YAP1 inhibition could replicate the tumor-suppressive effect of SUNE1 and CNE1 cells. Our results showed that cell growth and colony formation ability in NPC cells were significantly inhibited when YAP1 knockdown (Figure 2A and B, ${ }^{*} \mathrm{p}<0.05$ ). And Wound-healing and invasion assays showed that SUNE1 and CNE1 cells migrated at a significantly slower rate when YAP1 levels were decreased through RNA interference comparing to the control groups (Figure $2 \mathrm{C},{ }^{*} \mathrm{p}<0.05$, $* * p<0.01)$. SUNE1 and CNE1 cells had fewer invasions in the lower chamber after transfected with YAP siRNA, and the invasion rate of each cell was $50 \%$ lower compared to its
A

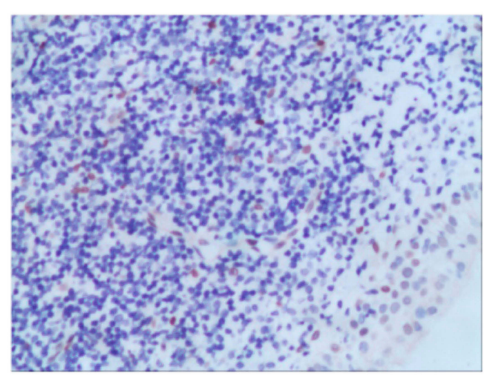

Normal

B

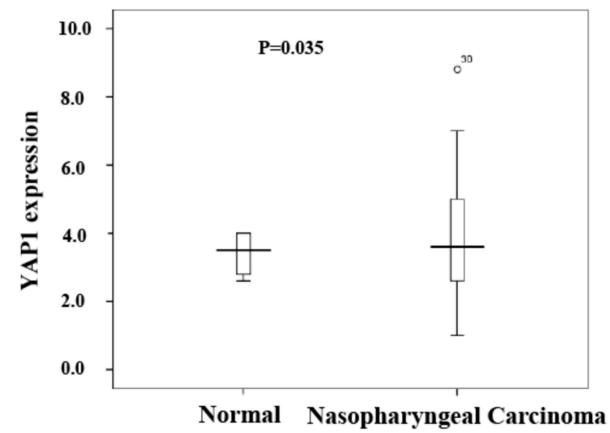

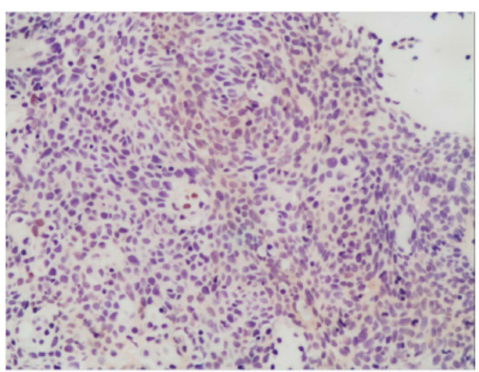

NPC

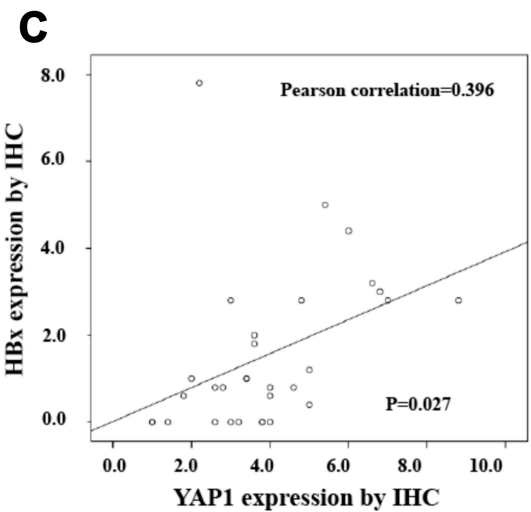

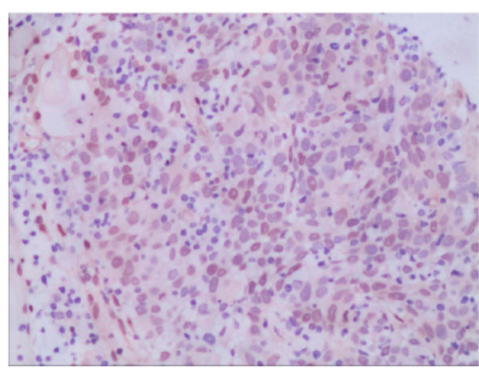

NPC+HBsAg (+)

D

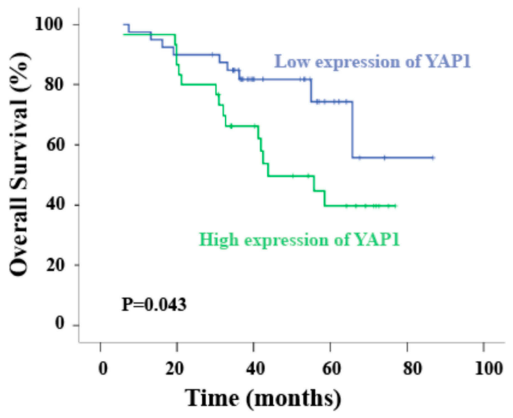

Figure I High YAPI expression in NPC tissue with HBV infection correlated with poor prognosis. 

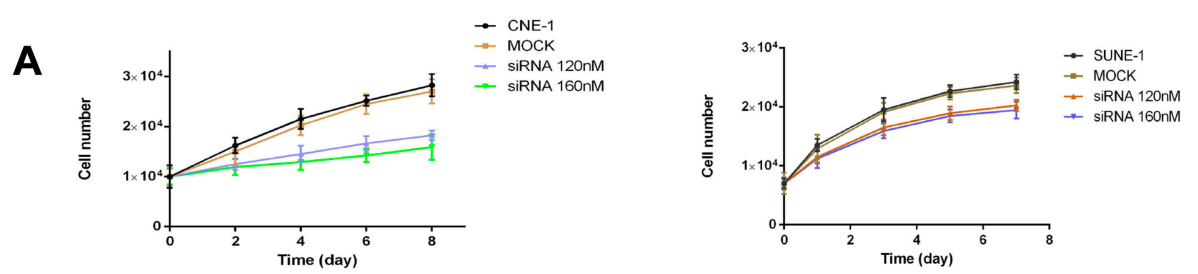

B
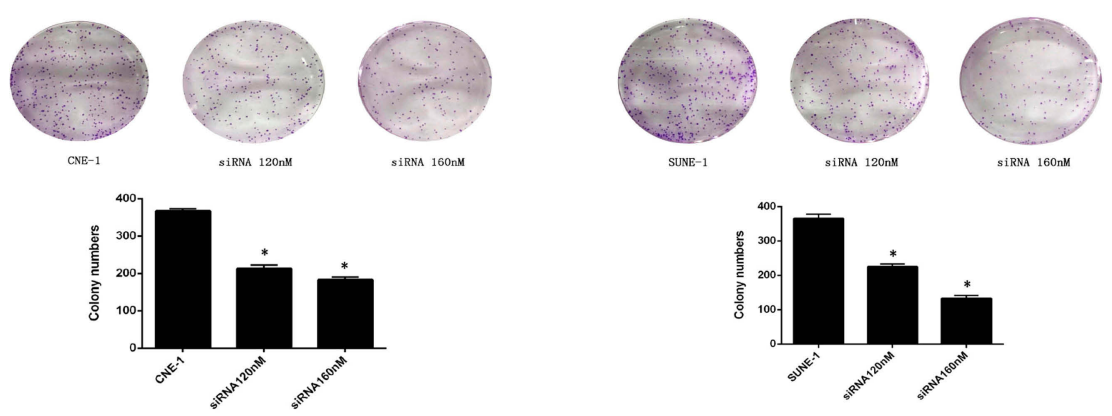

C
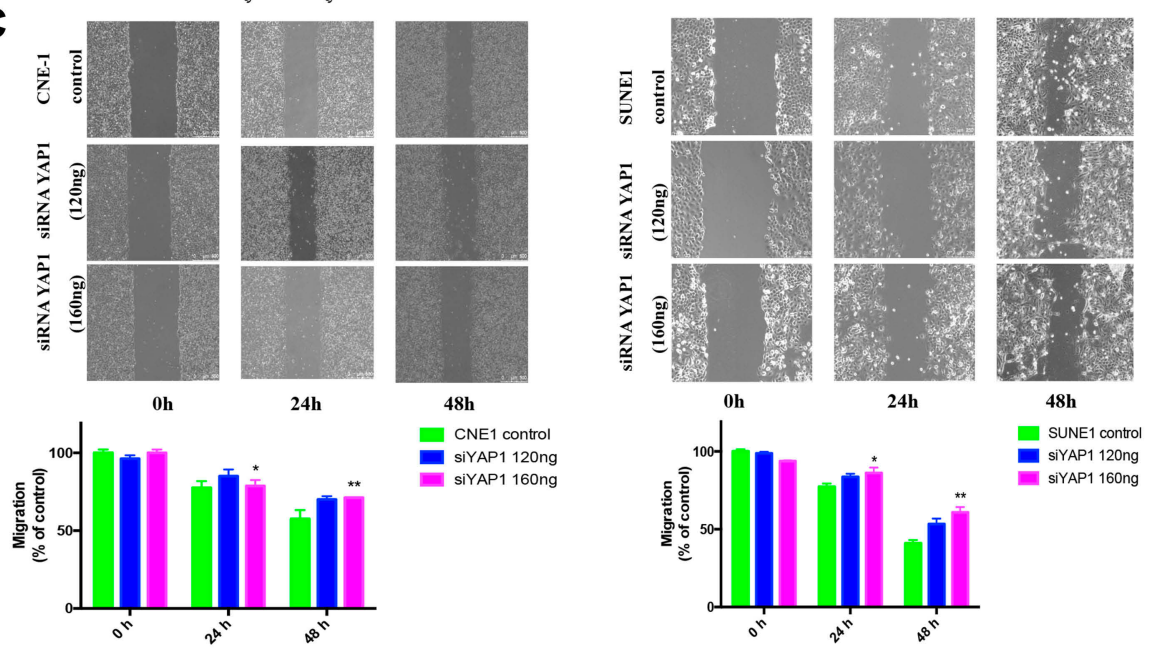

D
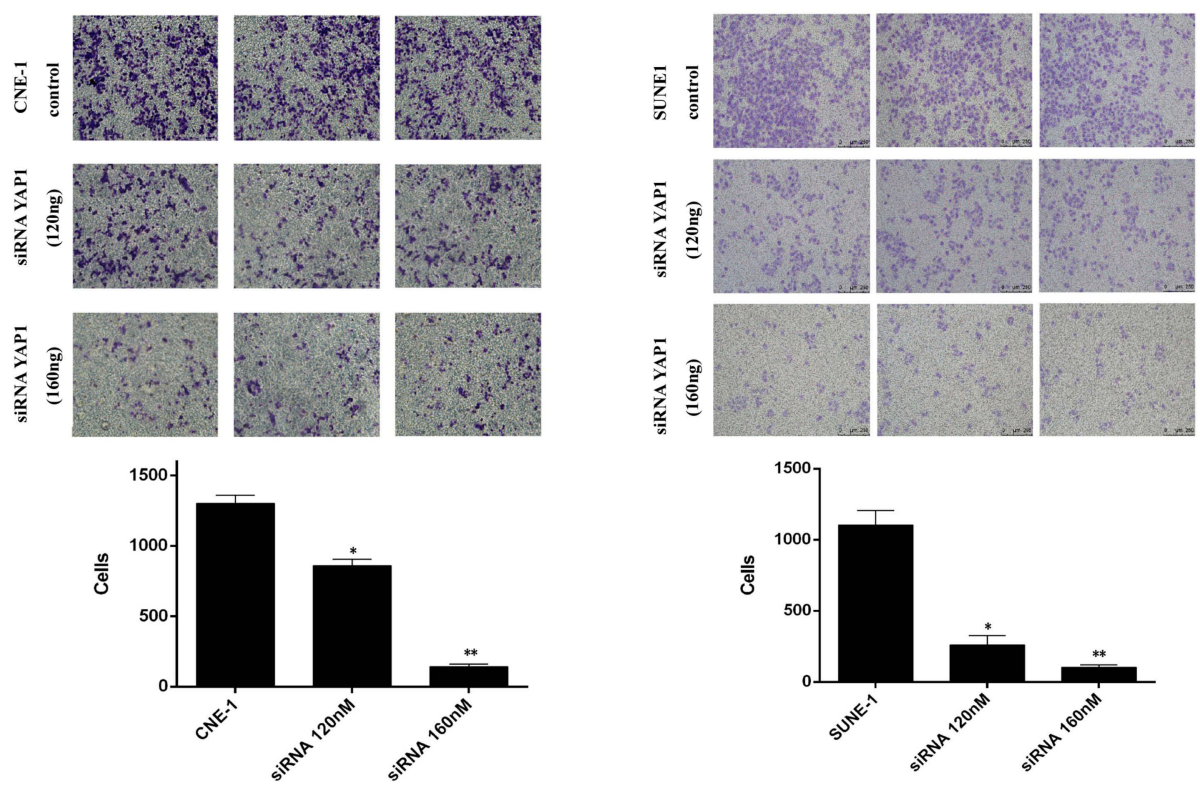

Figure 2 YAPI inhibition reduced NPC cell proliferation, migration and invasion. 
control group (Figure 2D, ${ }^{*} \mathrm{p}<0.05,{ }^{* *} \mathrm{p}<0.01$ ). These results proved that YAP1 inhibition could reduce NPC cell proliferation, migration, and invasion, which indicate that targeting YAP1 could suppress NPC growth and invasion.

\section{YAPI Promotes Tumor Metastasis Through Regulating the EMT Pathway}

To further explore the pathway regarding YAP1 mediated NPC metastasis, we evaluated EMT related markers by performing PCR and Western blot analyses. The expression levels of EMT key regulators (LATS1, MST1, Snail, E-cadherin, and N-cadherin) were detected after NPC cells were treated with siYAP1 or YAP1 DNA plasmid. With the depletion of YAP1, MST1 and E-cadherin increased, while LATS1 decreased in both CNE-1 and SUNE1 cell lines, and $\mathrm{N}$-cadherin decreased were detected only in CNE1 cell, E-cadherin and Snail increased only in SUNE1 cell line (Figure $3 \mathrm{~A}$ and $\mathrm{B},{ }^{*} \mathrm{p}<0.05,{ }^{*} \mathrm{p}<0.01$ ). Meanwhile, as YAP1 exogenously increased (Figure 3C and D), MST1 and E-cadherin expressions were decreased in CNE1cell; however, LATS1 still decreased in both CNE1 and SUNE1 cell lines, and Snail expression showed no marked difference between the two treatments. Nevertheless, differential expression of the EMT markers, after alteration of YAP1, indicates that the regulatory role of YAP1 in NPC metastasis may be through EMT.

\section{HBx, Correlated with YAPI, Promotes Tumor Proliferation, Migration and Invasion}

In our previous study, we had found that HBsAg (+) patients account for $10.3 \%$ of the NPC group and had a poor survival rate if the HBsAg $(+)$ patient showed a high level of pretreated EBV DNA; therefore, we explored the role of HBx in NPC cell lines. We found that HBx plasmid transfection could promote cell growth and colony formation in SUNE1 and CNE1 cells, even after treatment with YAP1 siRNA (Figure 4A and B, $\left.{ }^{*} \mathrm{p}<0.05, * * \mathrm{p}<0.01\right)$. Interestingly, this procedure could restore NPC cell's YAP1 expression. By using an in vitro invasion and migration assay, we also found a significant increase in the rate of NPC cell invasion and migration under these conditions (Figure $4 \mathrm{C},{ }^{*} \mathrm{p}<0.05,{ }^{*} \mathrm{p}<0.01$ ). Additionally, with an increase of HBx, YAP1 was highly expressed (Figure 5A), while transfected HBx with siYAP1 together, LATS1 decreased, and MST1 increased in both cell lines, but Snail increased only in SUNE1; however, E-cadherin and $\mathrm{N}$-cadherin expression showed no difference in CNE1 and SUNE1 (Figure 5B and C, $\left.{ }^{*} \mathrm{p}<0.05,{ }^{*} \mathrm{p}<0.01\right)$. Notably, HBx promoted NPC cell invasion through EMT, which correlated with YAP1. The effects of HBx and YAP1 moderation suggest that targeting of HBx and/or YAP1 could regulate NPC growth, invasion and migration.

\section{Anti-YAPI Inhibits Tumor Cell Metastasis in vivo, and $\mathrm{HBx}$ Directly Up-Regulates YAPI Expression Through Binding to Its \\ Promoter Region}

We established metastasis in a mouse model by injecting sh-YAP1 SUNE1 cells or control shRNA (with or without HBx DNA plasmid, for each group, respectively) into nude mice's tail veins, and found lung metastasis colonization was remarkably suppressed in both the shYAP1 and shYAP1 with HBx groups, only one in six mice was found, respectively, and in the vehicle group, three in six mice were found with lung metastasis colonization, while treatment with $\mathrm{HBx}$ found four in six mice with lung colonization sites (Figure 6A and B). We performed IHC analysis for vimentin and E-cadherin expression of the lung metastasis colonization site of mice, vimentin and E-cadherin expression were decreased in ShYAP1 group compared to the vehicle group, while with HBx exogenously increase could enhance vimentin and E-cadherin expression significantly; however, in ShYAP1 with HBx exogenously increase group, vimentin and E-cadherin expression showed no such increasing level compared to the vehicle with HBx group (Figure 6C). Thus, we concluded that HBx could induce metastasis, while anti-YAP1 could inhibit tumor cell metastasis in vivo.

To further delineate the interaction of HBx with YAP1, we performed a Dual-Luciferase reporter assay. Literature indicated that the YAP promoter was located at nt 2232/ $1115{ }^{15}$ Thus, we attempted to confirm this in our model. Cotransfection of HBx expression plasmid (pCMV-X, 0.2 ng/well), pGL3-YAP1 or pGL3-Basic control (0.2 ng/well) was performed in SUNE-1 cells. Relative luciferase activity of pGL3-YAP1 (pGL3-348) increased by 5- or 10-fold on average by HBx in the HBx and YAP1 gene cotransfection group. However, the pGL3-Basic control construct was not activated by HBx, let alone the pGL3-Basic control or pGL3-YAP1 (pGL3-348) group (Figure 6D). Consistent with the study before, ${ }^{15}$ we proved that the regulation site of HBx is located -348 of YAP1 in NPC 
A
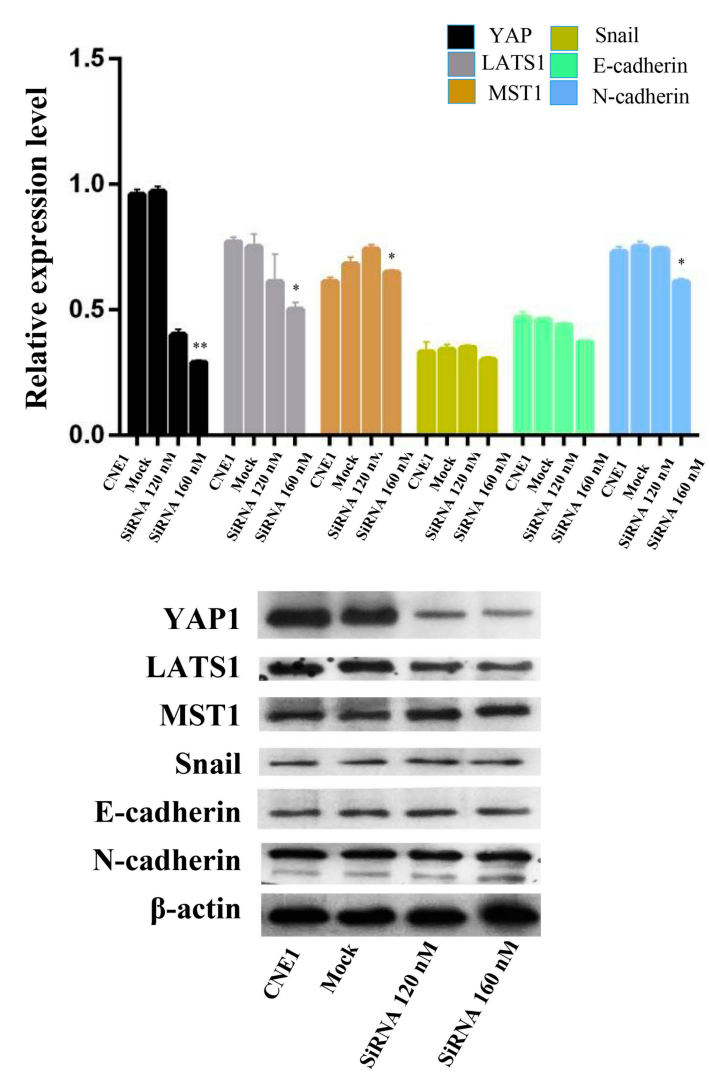

C
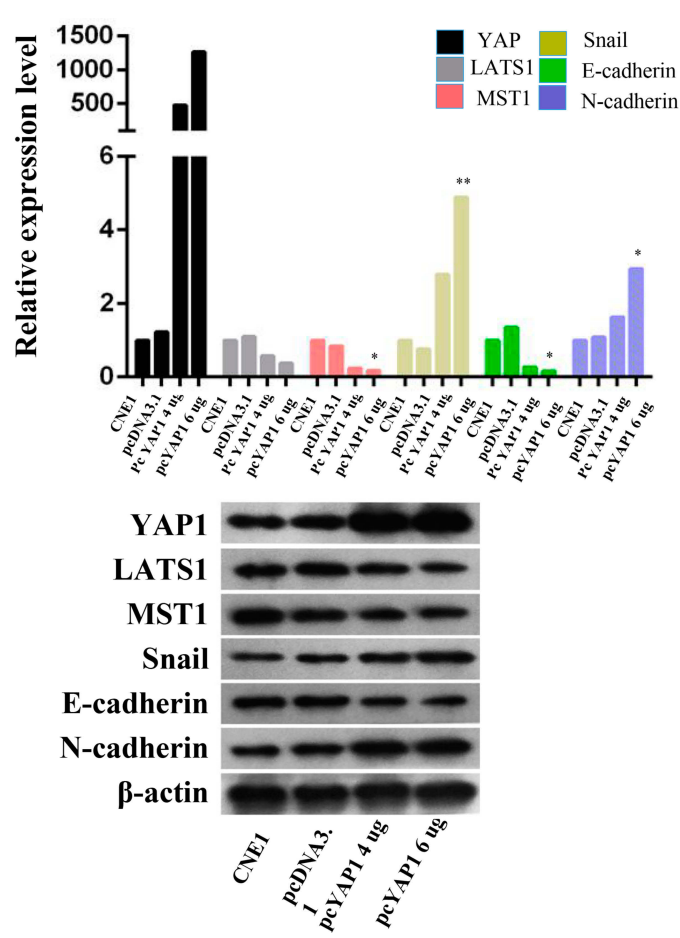

Figure 3 YAPI promotes NPC metastasis by regulating the EMT pathway.
B
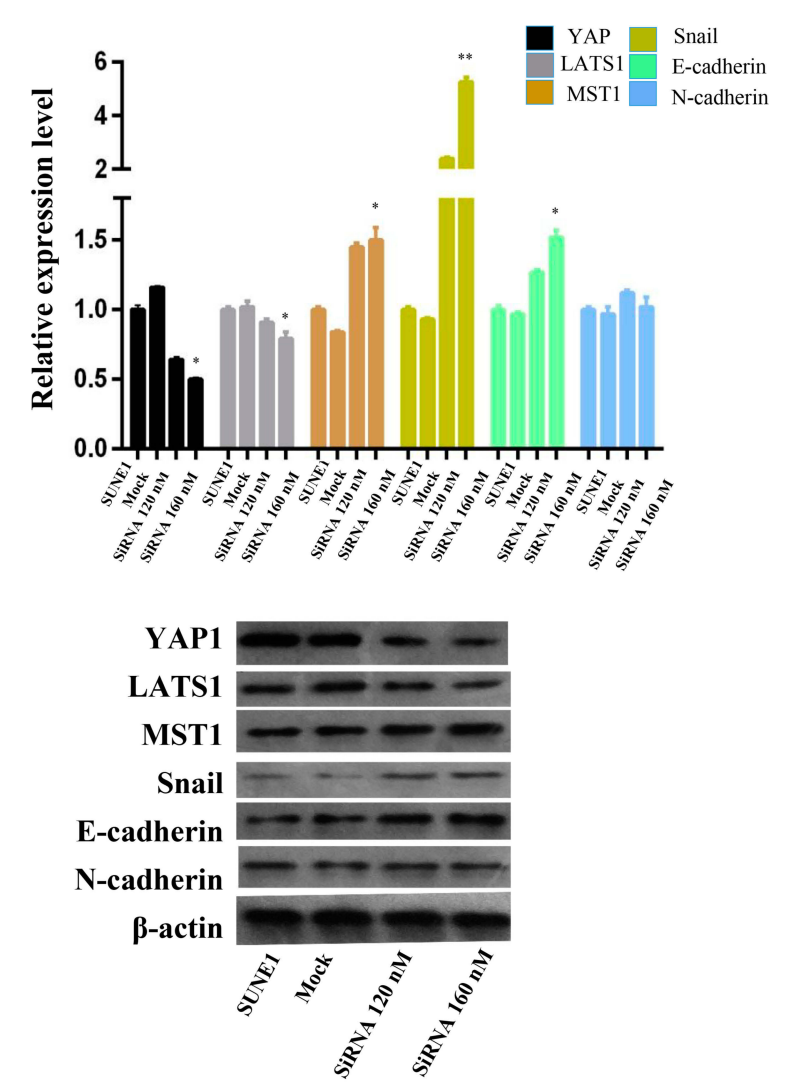

D
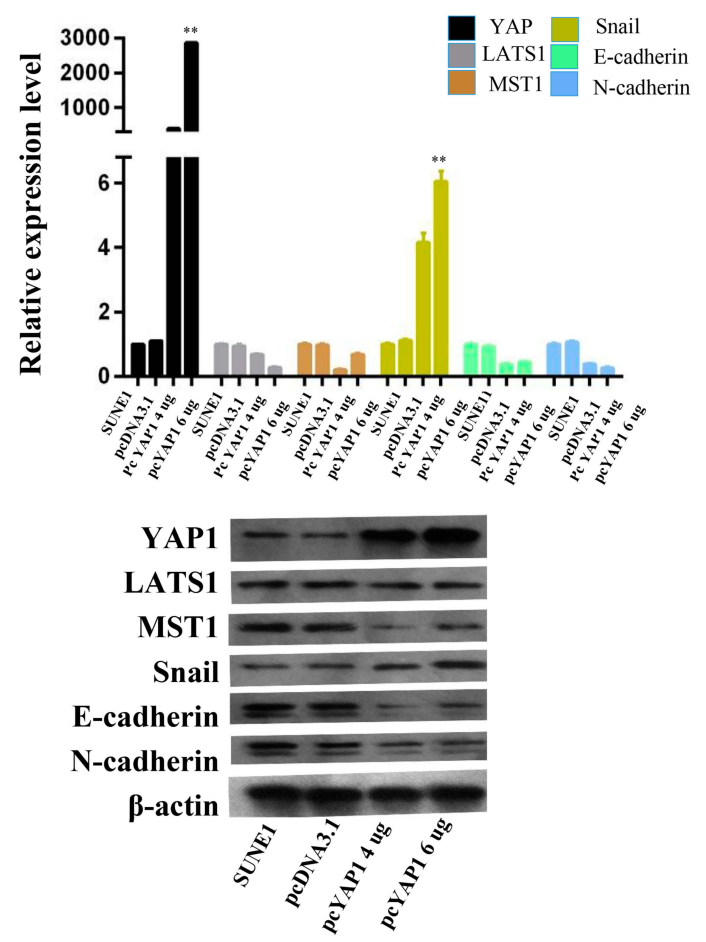
A

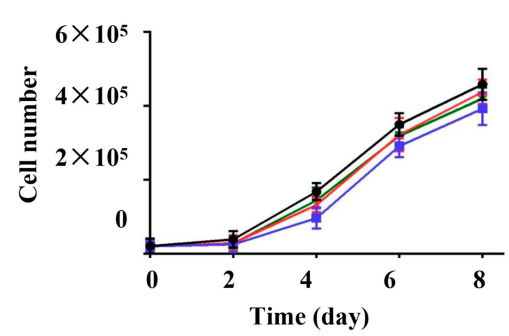

B

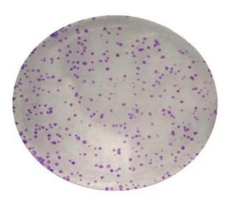

siRNA

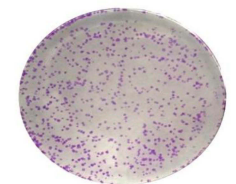

SiRNA + pHBX

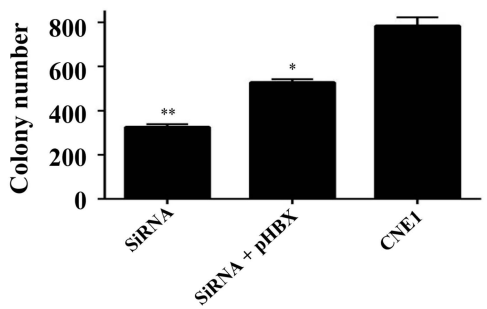

C
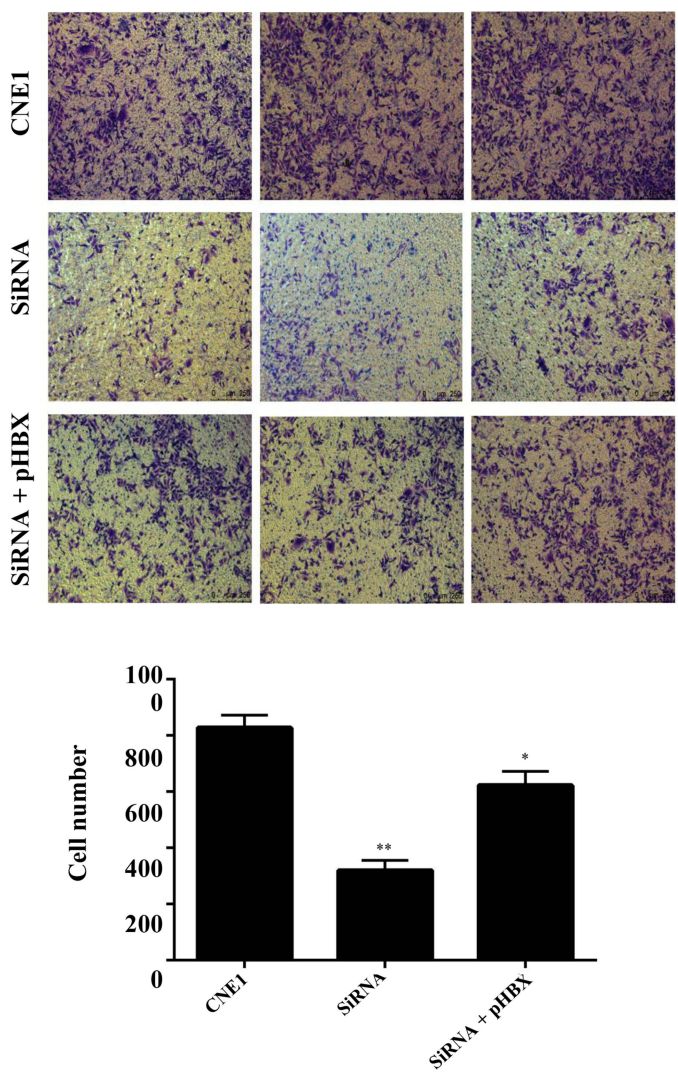

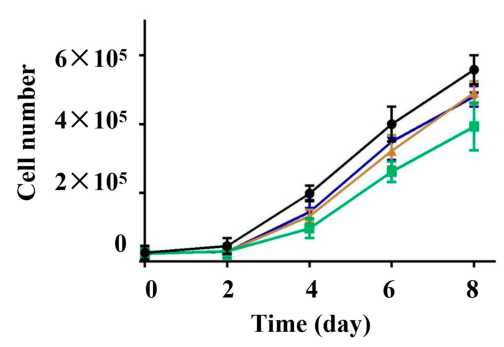

$\rightarrow$ SUNE1

$\rightarrow$ SiRNA

$\rightarrow$ SiRNA + pHBX

$\rightarrow$ SiRNA + pHBX2

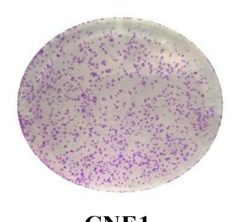

CNE1

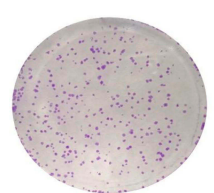

siRNA

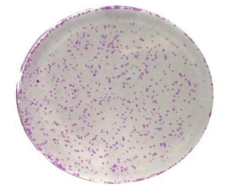

SiRNA + pHBX

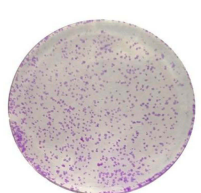

SUNE1
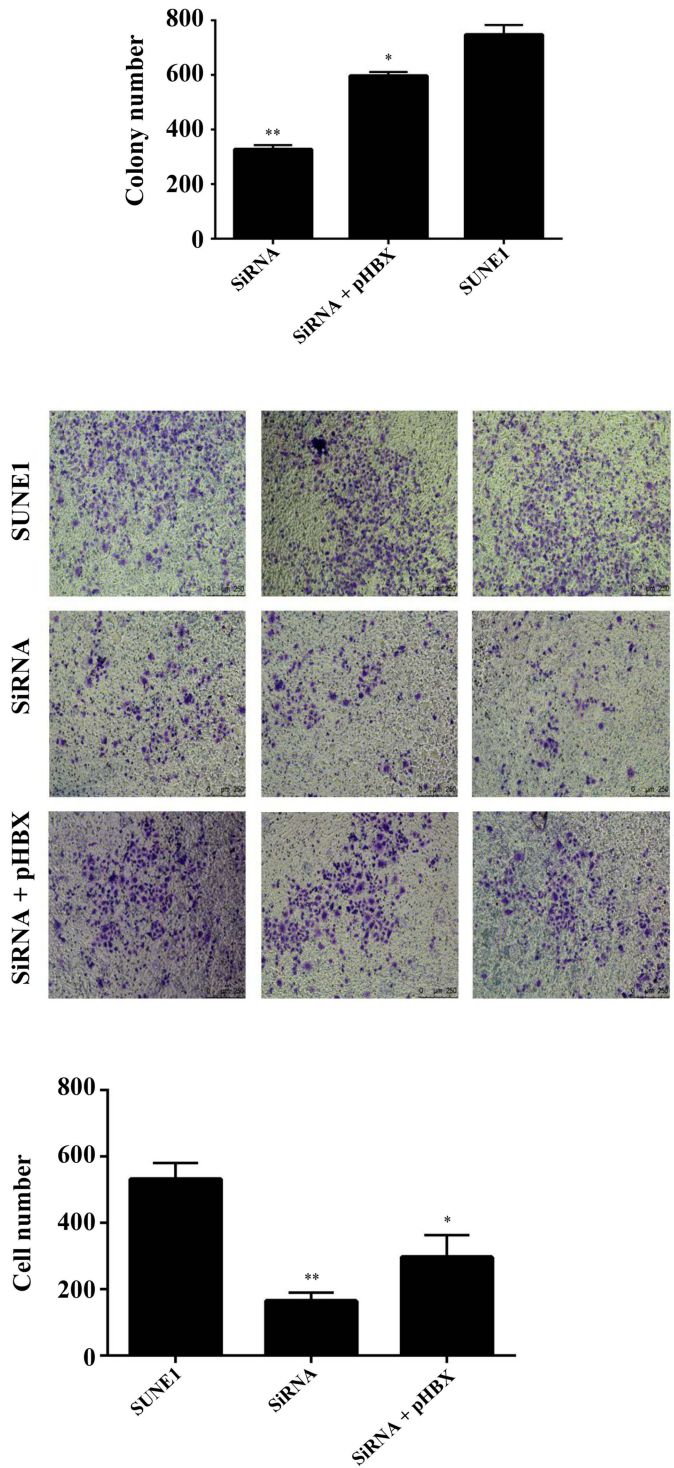

Figure $4 \mathrm{HBx}$ promotes NPC cell proliferation, migration and invasion, correlated to YAPI. 
A

CNE1

YAP1

$\beta$-Actin

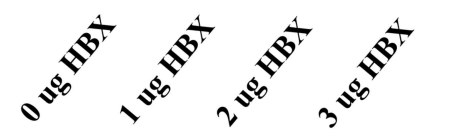

B

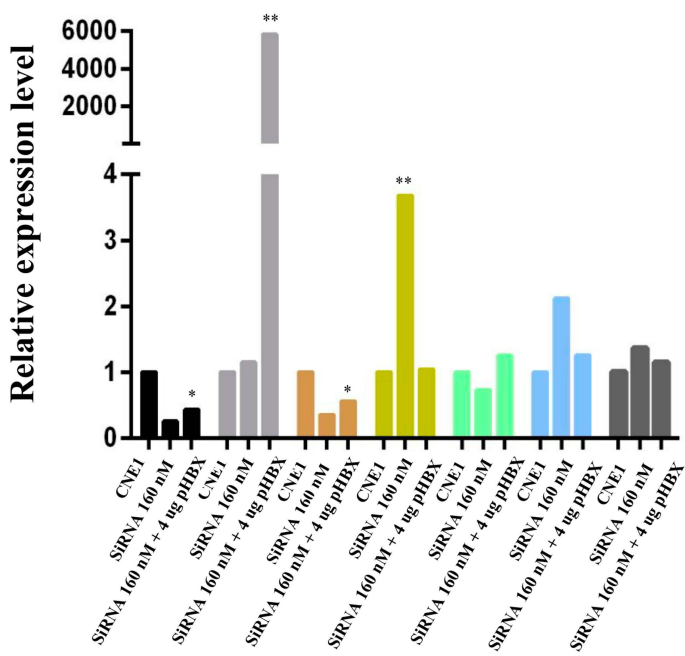

C

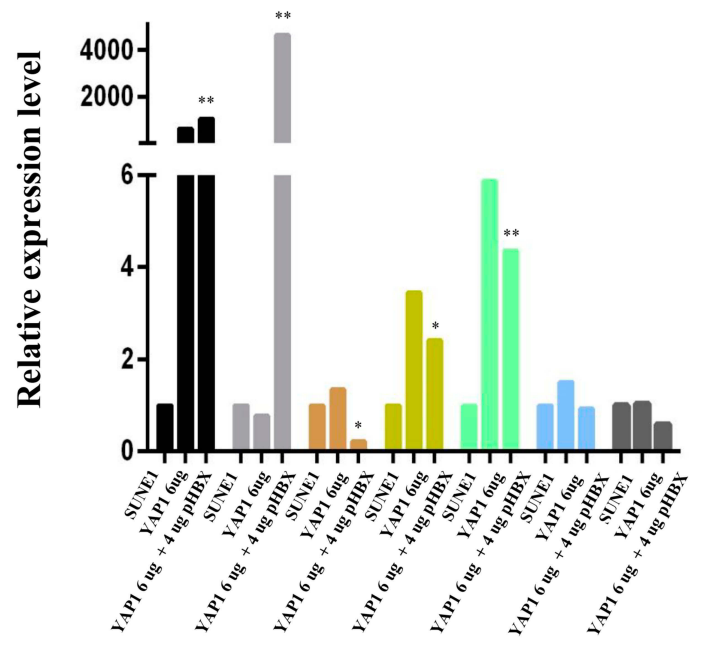

SUNE1

YAP1

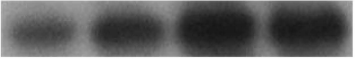

$\beta$-Actin

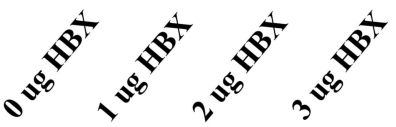

YAP1

HBX

LATS1

MST1

Snail

E-cadherin

$\mathrm{N}$-cadherin

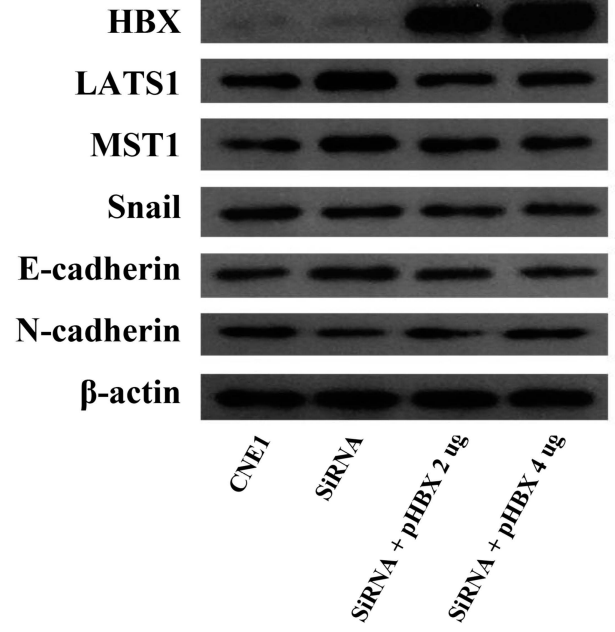

\begin{tabular}{ll}
\hline HBX \\
LATS1 \\
MST1 \\
Snail \\
E-cadherin \\
N-cadherin
\end{tabular}

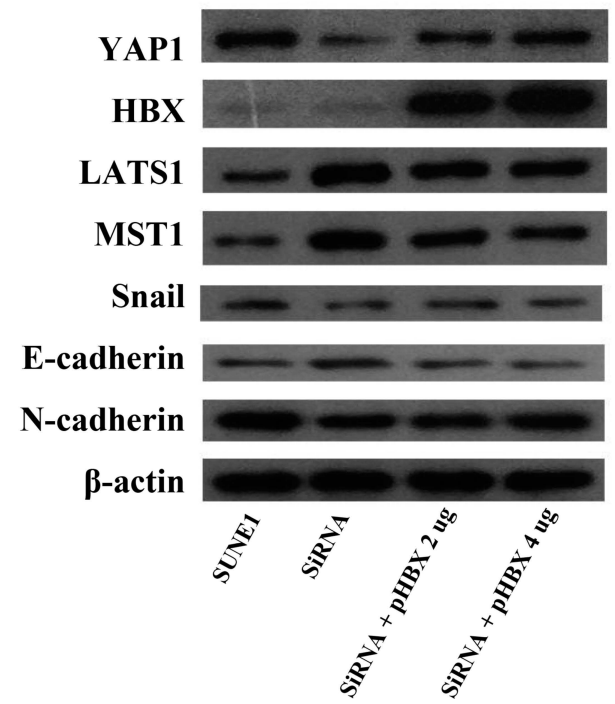

Figure $5 \mathrm{HBx}$ regulate YAPI expression and EMT. 
A
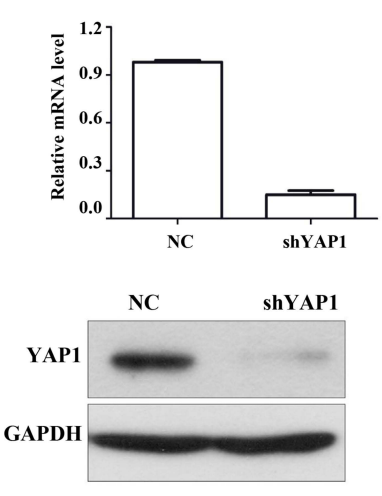

C
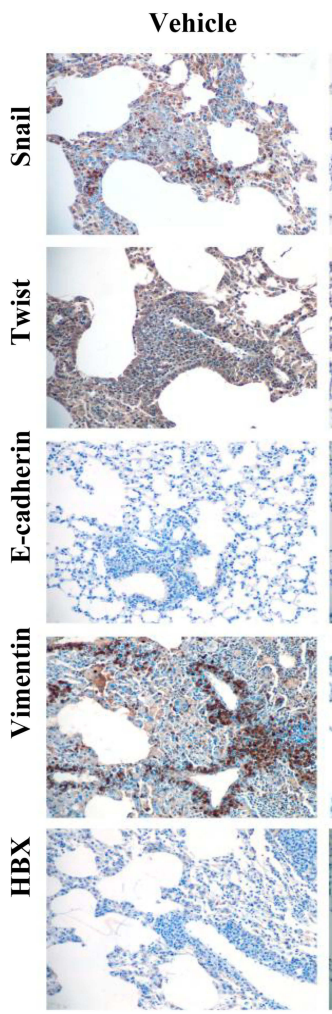

B
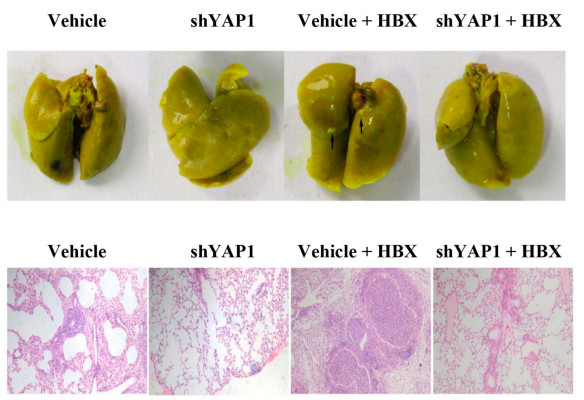

\begin{tabular}{llc}
\hline \multicolumn{1}{c}{ Group } & Mice & Lung-metastasis \\
\hline Vehicle & $\mathrm{n}=6$ & 3 \\
shYAP1 & $\mathrm{n}=6$ & 1 \\
Vehicle +HBX & $\mathrm{n}=6$ & 4 \\
shYAP1 + HBX & $\mathrm{n}=6$ & 1 \\
\hline
\end{tabular}

D

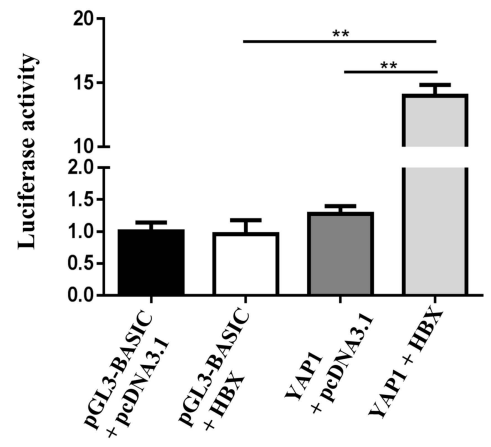

shYAP1 $\quad$ Vehicle + HBX $\quad$ shYAP1 + HBX
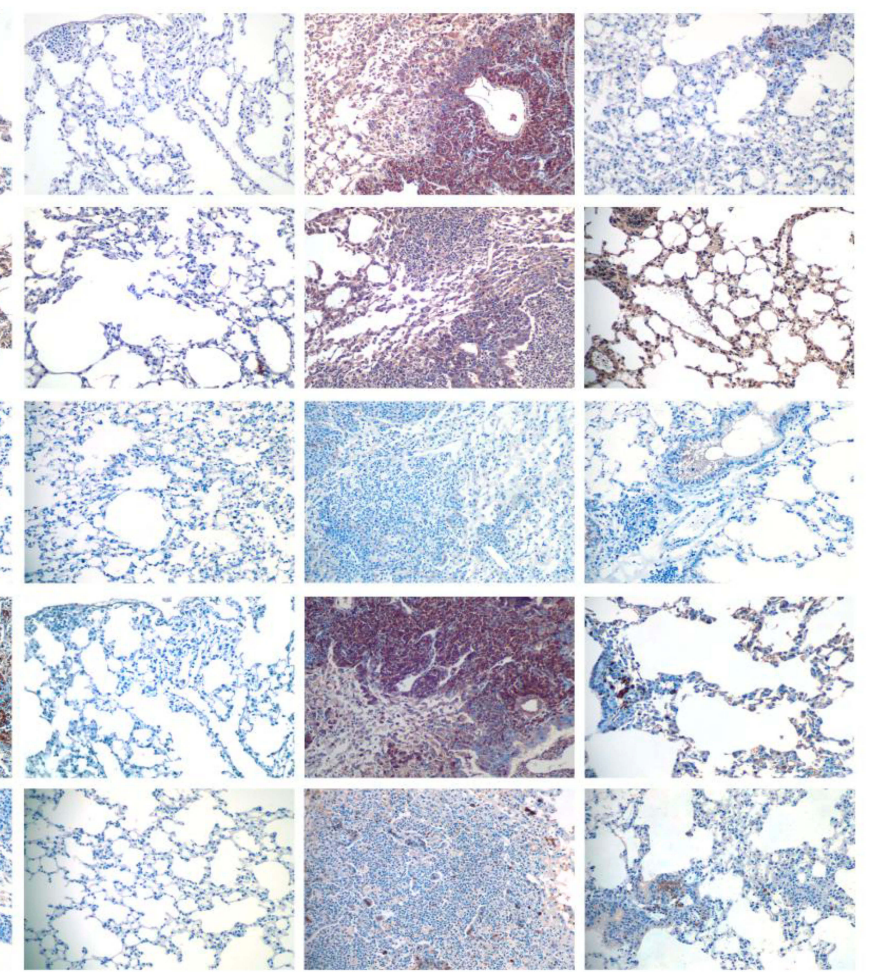

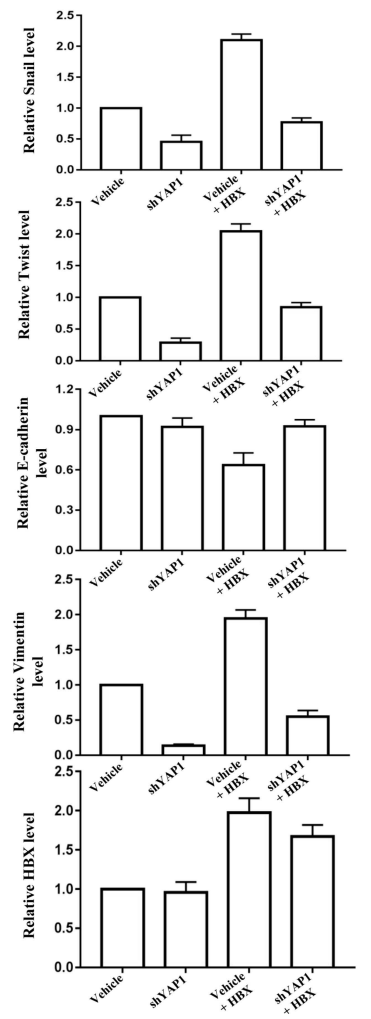

Figure 6 Anti-YAPI inhibits tumor cell metastasis in vivo, and HBX directly up-regulates YAPI expression through binding to its promoter region.

cell. Collectively, we concluded that YAP1 is a driving factor in NPC cell metastasis, and HBx plays a promoting role in this process, but the interaction between these two markers needs further investigation.

\section{Discussion}

Human viral infection has increasingly been established as playing a significant role in the hallmarks of cancer oncogenesis. ${ }^{19,20}$ Within the endemic area of NPC, EBV associated non-keratinizing squamous cell carcinoma has accounted for approximately $95 \%$, and interestingly, $10.3 \%$ of patients were infected with chronic HBV. ${ }^{9}$ However, its molecular mechanism remains unknown. In the current study, we first revealed a higher YAP1 expression in NPC with HBV infection, and showed that HBx could regulate YAP1, which further increasing NPC cell invasion through the EMT process. Our findings demonstrate that YAP1 could be used as a promising prognostic and treatment target for NPC with HBV infection. 
YAP1, a key downstream effector of the Hippo pathway, functions as a transcriptional co-activator. YAP1 is highly expressed in various types of cancers, which could promote cell proliferation and invasion. Evidence indicates that YAP1 has great clinical significance. According to TCGA data analyses, its expression was correlated with survival in gastric, lung, colorectal and hepatocellular cancers. ${ }^{10}$ YAP1 could drive cell proliferation in oral squamous cell carcinoma ${ }^{21}$ and promote glycolysis in the progression of NPC. ${ }^{22}$ ONCOMINE database analysis confirmed that gene expression of YAP1 was increasingly associated with $\mathrm{T}$ advanced and $\mathrm{N}$ advanced staged NPC tissue, as Supplementary Figure 2 data shown. High expression of YAP1 was closely associated with poor survival, based on our previous RPPA proteomic profiling, ${ }^{14}$ which we confirmed in the current study by using IHC to determine YAP1 and HBx differential protein expression and clinical prognosis within the patient sample groups. As expected, cell proliferation, migration and invasion were decreased when YAP1 expression inhibited. These results suggest that YAP1 is a major driver protein in NPC metastasis. However, the mechanism underlying the promotion of NPC metastasis by YAP1 has not yet been elucidated, which leads us for further investigation.

The Hippo pathway is crucial for organ development and homeostasis maintenance. The key members of the mammalian Hippo pathway include a kinase cascade of mammalian sterile 20-like kinase 1/2 (MST1/2) and large tumor suppressor kinase 1/2 (LATS1/2). ${ }^{2,24}$ MST1/2 interact with and phosphorylate LATS1/2 kinase. Active LATS1/2 kinase inactivates YAP1 and PDZ-binding motif (TAZ) to affect their localization. ${ }^{25,26}$ Considering YAP1 is a key component of Hippo pathway, and its important function in homeostasis maintenance, as shown in our results, YAP1 expression could influence MST1/2 and LATS1/2, which lead us to wonder how YAP1 function in homeostasis maintenance. It is reported that YAP1 promotes tumor proliferation by targeting important genes including Ki-67, SOX4, H19 and c-MYC, the latter one was positively related to EMT. ${ }^{27}$ Our RPPA data also indicated that YAP1 was strongly correlated with c-MYC expression (Supplementary Figure 1D), which is consistent with those studies. Indeed, we showed that anti-YAP1 inhibits NPC cell migration and invasion by regulating EMT. To further investigate the underlying mechanisms, we attempted to identify potential targets of YAP1 in regulating NPC metastasis. $\mathrm{HBx}$ reportedly plays an important role in hepatocellular carcinoma and can upregulate YAP1 expression. ${ }^{15,16}$ Interestingly, a similar phenomenon of HBx regulating YAP1 has also been observed in NPC cell lines. We confirmed that YAP1 was a direct target of HBx by using luciferase reporter assay, which could prove our hypothesis. Thus, we can further conclude that YAP1 induces cell invasion and migration through the EMT axis, which could be mediated by HBx.

During tumor progression, EMT could trigger aggressive features including cell migration, invasion, and treatment resistance. ${ }^{28}$ EMT is a factor in cancer metastasis. ${ }^{29}$ Hallmarks of EMT are including loss of E-cadherin, increase of mesenchymal and extracellular matrix biomarkers N-cadherin, and vimentin. ${ }^{30,31}$ Varieties of signaling pathways are involved with EMT regulation, including TGF- $\beta /$ Snail, GSK3 $\beta$, EGFR, and ERK/MAPK. ${ }^{32}$

It is reported that YAP/TAZ signaling plays an important role in EMT regulation. YAP/TAZ activation could promote cell proliferation and EMT. ${ }^{33}$ YAP1 could activate Slug and vimentin-mediated EMT with the transcription factor FOS. ${ }^{13}$ Weiss et al found that Snail/Slug-YAP/TAZ complexes could modulate self-renewal and cell differentiation. ${ }^{34}$ Crosstalk between YAP/TAZ and EMT transcription factors like Snail/Slug and Twist could promote cancer cell EMT process, which further acquires malignant features.

In our study, we showed that anti-YAP1 inhibits cell migration and invasion, and with E-cadherin highly reexpressed, while LATS1 and MST1 showed a little decrease. However, when YAP1 expression exogenously increased, we found that E-cadherin expression loss, LATS1 and MST1 decreased, together with Snail expression level increasing. All those evidence lead us to conclude that YAP1 promotes metastasis by regulating EMT in NPC cells. Further, we had identified HBx is the potential target of YAP1 in regulating NPC metastasis. HBx has been reported to take an important role in hepatocellular carcinoma, and it could up-regulate YAP1 expression. ${ }^{15,16,35,36}$ HBx could dysfunction intercellular adhesion, and induce EMT and metastasis in HCC. Research showed that HBx could induce HMGA2 by regulating EMT, subsequently leading to HCC cell invasion. ${ }^{37}$ These evidence suggest that HBx might regulate YAP1 in the EMT process. Indeed, we proved that $\mathrm{HBx}$ regulated promoter region of YAP was located at nt -348 through luciferase reporter gene assays. And with HBx increasing expression, NPC cell invasion and migration ability enhanced. Our study firstly reported that YAP1 induce cell invasion and migration through EMT axis, and could be mediated by HBx 
activity in NPC, which could provide a new strategy for NPC metastasis clinical treatment thinking.

In summary, the present study demonstrated that YAP1 promotes metastasis through the regulation of the EMT axis which can be induced by $\mathrm{HBx}$. Our findings offer new therapeutic approaches for NPC treatment and serve as a promising prognosis and treatment target for NPC patients with $\mathrm{HBV}$ infection.

\section{Acknowledgment}

Zeli Huang, Bojin Su, and Fang Liu are co-first authors in this study.

\section{Funding}

This work was financially supported by grants from the National Natural Science Foundation of China (No.81402244 [to Ying Huang] and 81602392 [to Tao Xu]), a Special Fund Project for 2016 University of Texas MD Anderson cancer center sister hospital network fund (SINF).

\section{Disclosure}

The authors report no conflicts of interest in this work.

\section{References}

1. Chen W, Zheng R, Baade PD, et al. Cancer statistics in China, 2015. CA. 2016;66(2):115-132. doi:10.3322/caac.21338

2. Lee AW, Ng WT, Chan LL, et al. Evolution of treatment for nasopharyngeal cancer - success and setback in the intensity-modulated radiotherapy era. Radiother Oncol. 2014;110(3):377-384. doi:10.10 16/j.radonc.2014.02.003

3. Chen YP, Chan ATC, Le QT, Blanchard P, Sun Y, Ma J. Nasopharyngeal carcinoma. Lancet. 2019;394(10192):64-80. doi:10. 1016/S0140-6736(19)30956-0

4. Tsao SW, Tsang CM, To KF, Lo KW. The role of Epstein-Barr virus in epithelial malignancies. J Pathol. 2015;235(2):323-333. doi:10. 1002/path. 4448

5. Chan AT. Nasopharyngeal carcinoma. Ann Oncol. 2010;21(Suppl 7): vii308-312. doi:10.1093/annonc/mdq277

6. Te HS, Jensen DM. Epidemiology of hepatitis B and C viruses: a global overview. Clin Liver Dis. 2010;14(1):1-21, vii. doi:10. 1016/j.cld.2009.11.009

7. Arzumanyan A, Reis HM, Feitelson MA. Pathogenic mechanisms in HBV- and HCV-associated hepatocellular carcinoma. Nat Rev Cancer. 2013;13(2):123-135. doi:10.1038/nrc3449

8. Liu X, Li X, Jiang N, et al. Prognostic value of chronic hepatitis $B$ virus infection in patients with nasopharyngeal carcinoma: analysis of 1301 patients from an endemic area in China. Cancer. 2014;120 (1):68-76. doi:10.1002/cncr.28377

9. Xu T, Huang Z, Deng Y, et al. Clinical implications of hepatitis $\mathrm{B}$ viral infection in Epstein-Barr virus-associated nasopharyngeal carcinoma. J Clin Virol. 2015;64:64-71. doi:10.1016/j.jcv.2014.11. 024

10. Poma AM, Torregrossa L, Bruno R, Basolo F, Fontanini G. Hippo pathway affects survival of cancer patients: extensive analysis of TCGA data and review of literature. Sci Rep. 2018;8(1):10623. doi:10.1038/s41598-018-28928-3
11. Shao DD, Xue W, Krall EB, et al. KRAS and YAP1 converge to regulate EMT and tumor survival. Cell. 2014;158(1):171-184. doi:10.1016/j.cell.2014.06.004

12. Omori H, Sato K, Nakano T, et al. Stress-triggered YAP1/SOX2 activation transcriptionally reprograms head and neck squamous cell carcinoma for the acquisition of stemness. $J$ Cancer Res Clin Oncol. 2019;145(10):2433-2444. doi:10.1007/s00432-019-02995-Z

13. Vincent-Mistiaen Z, Elbediwy A, Vanyai H, et al. YAP drives cutaneous squamous cell carcinoma formation and progression. Elife. 2018;20:7.

14. Xu T, Su B, Huang P, et al. Novel biomarkers of nasopharyngeal carcinoma metastasis risk identified by reverse phase protein array based tumor profiling with consideration of plasma Epstein-Barr virus DNA load, Proteomics. Clin Appl. 2017;11(9-10). doi:10.1002/prca.201700015

15. Zhang T, Zhang J, You X, et al. Hepatitis B virus X protein modulates oncogene yes-associated protein by CREB to promote growth of hepatoma cells. Hepatology. 2012;56(6):2051-2059. doi:10.1002/ hep. 25899

16. Wang Y, Fang R, Cui M, et al. The oncoprotein HBXIP up-regulates YAP through activation of transcription factor c-Myb to promote growth of liver cancer. Cancer Lett. 2017;385:234-242. doi:10.10 16/j.canlet.2016.10.018

17. Pan Y, Wang S, Su B, et al. Stat3 contributes to cancer progression by regulating Jab1/Csn5 expression. Oncogene. 2017;36(8):1069-1079. doi:10.1038/onc.2016.271

18. Wang S, Pan Y, Zhang R, et al. Hsa-miR-24-3p increases nasopharyngeal carcinoma radiosensitivity by targeting both the $3^{\prime}$ UTR and $5^{\prime}$ UTR of Jab1/CSN5. Oncogene. 2016;35(47):6096-6108. doi:10.1038/onc.2016.147

19. Mesri EA, Feitelson MA, Munger K. Human viral oncogenesis: a cancer hallmarks analysis. Cell Host Microbe. 2014;15 (3):266-282. doi:10.1016/j.chom.2014.02.011

20. Moore PS, Chang Y. Why do viruses cause cancer? Highlights of the first century of human tumour virology. Nat Rev Cancer. 2010;10 (12):878-889. doi:10.1038/nrc2961

21. Hiemer SE, Zhang L, Kartha VK, et al. A YAP/TAZ-Regulated molecular signature is associated with oral squamous cell carcinoma. $M C R$. 2015;13(6):957-968. doi:10.1158/1541-7786.MCR-14-0580

22. Song L, Tang H, Liao W, et al. FOXC2 positively regulates YAP signaling and promotes the glycolysis of nasopharyngeal carcinoma. Exp Cell Res. 2017;357(1):17-24. doi:10.1016/j.yexcr.2017.04.019

23. Callus BA, Verhagen AM, Vaux DL. Association of mammalian sterile twenty kinases, Mst1 and Mst2, with hSalvador via C-terminal coiled-coil domains, leads to its stabilization and phosphorylation. FEBS J. 2006;273(18):4264-4276. doi:10.1111/ j.1742-4658.2006.05427.x

24. Praskova M, Xia F, Avruch J. MOBKL1A/MOBKL1B phosphorylation by MST1 and MST2 inhibits cell proliferation. CB. 2008;18 (5):311-321. doi:10.1016/j.cub.2008.02.006

25. Huang J, Wu S, Barrera J, Matthews K, Pan D. The Hippo signaling pathway coordinately regulates cell proliferation and apoptosis by inactivating Yorkie, the Drosophila Homolog of YAP. Cell. 2005;122 (3):421-434. doi:10.1016/j.cell.2005.06.007

26. Ren F, Zhang L, Jiang J. Hippo signaling regulates Yorkie nuclear localization and activity through 14-3-3 dependent and independent mechanisms. Dev Biol. 2010;337(2):303-312. doi:10.1016/j.ydbio.20 09.10 .046

27. Luo G, Hao NB, Hu CJ, et al. HBV infection increases the risk of pancreatic cancer: a meta-analysis. CCC. 2013;24(3):529-537. doi:10.1007/s10552-012-0144-2

28. Zanconato F, Forcato M, Battilana G, et al. Genome-wide association between YAP/TAZ/TEAD and AP-1 at enhancers drives oncogenic growth. Nat Cell Biol. 2015;17(9):1218-1227. doi:10.1038/ncb3216

29. Lamouille S, Xu J, Derynck R. Molecular mechanisms of epithelial-mesenchymal transition. Nat Rev Mol Cell Biol. 2014;15 (3):178-196. doi: $10.1038 / \mathrm{nrm} 3758$ 
30. Plaks V, Chou J, Maynard C, et al. Targeting matrix metalloproteinases (MMP) for anti-metastatic therapy: Blocking active MMP9 abrogates metastatic niche formation and prevents metastatic seeding in a breast cancer model. Cancer Res. 2015;75(21):4494-4503. doi:10.1158/0008-5472.CAN-14-3569

31. Gravdal K, Halvorsen OJ, Haukaas SA, Akslen LA. A switch from E-cadherin to $\mathrm{N}$-cadherin expression indicates epithelial to mesenchymal transition and is of strong and independent importance for the progress of prostate cancer. Clin Cancer Res. 2007;13(23):70 03-7011. doi:10.1158/1078-0432.CCR-07-1263

32. Gonzalez DM, Medici D. Signaling mechanisms of the epithelial-mesenchymal transition. Sci Signal. 2014;7(344):re8. doi:10.1126/scisignal.2005189

33. Lei QY, Zhang H, Zhao B, et al. TAZ promotes cell proliferation and epithelial-mesenchymal transition and is inhibited by the hippo pathway. Mol Cell Biol. 2008;28(7):2426-2436. doi:10.1128/ MCB.01874-07
34. Tang Y, Feinberg T, Keller ET, Li XY, Weiss SJ. Snail/Slug binding interactions with YAP/TAZ control skeletal stem cell self-renewal and differentiation. Nat Cell Biol. 2016;18(9):917-929. doi:10.1038/ ncb3394

35. Ha HL, Kwon T, Bak IS, Erikson RL, Kim BY, Yu DY. IGF-II induced by hepatitis $\mathrm{B}$ virus $\mathrm{X}$ protein regulates EMT via SUMO mediated loss of E-cadherin in mice. Oncotarget. 2016;7 (35):56944-56957. doi:10.18632/oncotarget.10922

36. Agostini A, Panagopoulos I, Andersen HK, et al. HMGA2 expression pattern and TERT mutations in tumors of the vulva. Oncol Rep. 2015;33(6):2675-2680. doi:10.3892/or.2015.3882

37. Zha Y, Yao Q, Liu JS, Wang YY, Sun WM. Hepatitis B virus $\mathrm{X}$ protein promotes epithelial-mesenchymal transition and metastasis in hepatocellular carcinoma cell line HCCLM3 by targeting HMGA2. Oncol Lett. 2018;16:5709-5714.

\section{Publish your work in this journal}

OncoTargets and Therapy is an international, peer-reviewed, open access journal focusing on the pathological basis of all cancers, potential targets for therapy and treatment protocols employed to improve the management of cancer patients. The journal also focuses on the impact of management programs and new therapeutic agents and protocols on patient perspectives such as quality of life, adherence and satisfaction. The manuscript management system is completely online and includes a very quick and fair peer-review system, which is all easy to use. Visit http://www.dovepress.com/ testimonials.php to read real quotes from published authors. 